\title{
The Feller Diffusion, Filter Rules and Abnormal Stock Returns
}

\author{
Paul Docherty ${ }^{\mathbf{a}}$, Yizhe Dong ${ }^{\mathbf{b}}$, Xiaojing Song ${ }^{\mathbf{c}}$ and Mark Tippett ${ }^{\mathbf{a}, \mathbf{d}, \text { * }}$ \\ a Business School, University of Newcastle, Callaghan, NSW, 2308, Australia \\ b Business School, University of Aberdeen, Aberdeen, AB24 3QY, UK; \\ c Business School, University of East Anglia, Chancellor's Drive, Norwich, NR4 7TJ, UK; \\ d Business School, University of Sydney, Codrington Street, Sydney, NSW, 2008, Australia
}

\begin{abstract}
:
We determine the conditional expected logarithmic (that is, continuously compounded) return on a stock whose price evolves in terms of the Feller diffusion and then use it to demonstrate how one must know the exact probability density that describes a stock's return before one can determine the correct way to calculate the abnormal returns that accrue on the stock. We show in particular that misspecification of the stochastic process which generates a stock's price will lead to systematic biases in the abnormal returns calculated on the stock. We examine the implications this has for the proper conduct of empirical work and for the evaluation of stock and portfolio performance.
\end{abstract}

Keywords: Feller diffusion; Fokker-Planck equation; Geometric Brownian Motion; Logarithmic return

JEL Classification: C52, G14, G17

Forthcoming European Journal of Finance

*Corresponding author. Email: M.Tippett@lboro.ac.uk. The authors acknowledge the helpful comments and suggestions of the Editor and referee. The usual disclaimer applies. 


\section{Introduction}

The Feller (1951a) diffusion (or continuous time branching process) has often been advanced as a model of stock price behaviour (Cox and Ross 1976; Cox 1996; Ashton et al. 2003, Gan and Waxman 2015). In this paper we determine the conditional expected logarithmic (that is, continuously compounded) return on a stock whose price evolves in terms of the Feller diffusion. We then use it to show that one must know the exact probability density that generates a stock's return before one can determine the correct way to calculate the abnormal returns that accrue on the stock. We show, for example, that if one mistakenly applies the abnormal returns formula for the Geometric Brownian Motion to a stock whose returns actually evolve in terms of the Feller diffusion, then there will be systematic biases in the computed abnormal returns which will give the appearance of abnormal returns when, in fact, there are no abnormal returns at all. Our analysis is purely theoretical in the sense that it contrasts key properties of two widely applied stochastic processes in the asset pricing literature and shows how they lead to contradictory specifications of the expected logarithmic returns that accrue on risky assets. We then develop the implications of this outcome for the way that abnormal stock returns ought to be calculated in the empirical work of the discipline. ${ }^{1}$

We commence our analysis in Section 2 by using the hyperbolic functions to model the evolution of stock prices in terms of a discrete time binomial filtration (Nelson and Ramaswamy 1990). Binomial models are particularly simple because they assume a stock's price will either increase with given probability or decline with complementary probability at each of the discrete points in time on which the modelling procedures are

\footnotetext{
${ }^{1}$ We emphasise in particular that we are not suggesting that the expected logarithmic returns formula for determining abnormal returns under the Feller diffusion is in any way superior to that of the equivalent formula for the Geometric Brownian Motion or any other stochastic process suitable for the modelling of stock prices.
} 
based. Moreover, variations in the stock's price and the probabilities associated with them can often be specified in such a way as to ensure that desired modelling requirements are satisfied - as, for example, when the stock's price cannot be negative or when increments in the stock's price must possess a mean and variance with given attributes. $^{2}$ We exploit this feature of the binomial filtration by specifying the discrete time mean and variance of increments in a stock's price in such a way as to ensure that as the time between stock price movements tends toward zero, the stock price itself will converge in distribution to the Feller diffusion. We then use the stochastic differential equation defined by these procedures to resolve an apparent inconsistency in the distributional properties of the Feller diffusion arising from the application of the Laplace transform to the Fokker-Planck (that is, Chapman-Kolmogorov) equation in Feller (1951b).

In Section 3 we broaden our use of the Fokker-Planck equation by determining the expected logarithmic return on a stock whose price evolves in terms of the Feller diffusion. We show, in particular, that the expected logarithmic return for the Feller diffusion varies according to the conditional probability of ultimate extinction (that is, the probability that the stock's price will eventually fall away to zero or equivalently, the firm will enter bankruptcy). This contrasts with the more commonly employed Geometric Brownian Motion for which the conditional expected logarithmic return is the same irrespective of the prevailing stock price. We demonstrate in particular, how the differing distributional properties of the Feller diffusion and the Geometric Brownian Motion will mean that it is crucially important for empirical researchers to test the compatibility of the observed returns on a given stock with the distributional assumptions on which the empirical analysis is based, before deciding on an appropriate quadrature

\footnotetext{
${ }^{2}$ This also makes binomial models particularly attractive because of the straightforward way in which they can be empirically implemented.
} 
formula through which to determine the abnormal returns that accrue on the stock. Section 4 concludes the paper.

\section{Feller Diffusion}

Let $x(t) \geq 0$ be a stock's price at time $t$. Now, suppose the increment in the stock's price over the period from time $t$ until time $(t+\Delta t)$ will be either positive, in the amount:

$$
\Delta x(t)=x(t+\Delta t)-x(t)=\sinh [\sigma \sqrt{x(t) \Delta t}]=\sigma \sqrt{x(t) \Delta t}+O\left[(\Delta t)^{3 / 2}\right]
$$

or negative, in the amount:

$$
\Delta x(t)=x(t+\Delta t)-x(t)=-\sinh [\sigma \sqrt{x(t) \Delta t}]=-\sigma \sqrt{x(t) \Delta t}+O\left[(\Delta t)^{3 / 2}\right]
$$

where $\sigma$ is a parameter whose meaning will become clear from context. Moreover, the probability of a positive increment in the stock's price is:

$$
\frac{1}{2}\left\{1+\tanh \left[\frac{\mu \sqrt{x(t) \Delta t}}{\sigma}\right]\right\}=\frac{1}{2}\left\{1+\frac{\mu \sqrt{x(t) \Delta t}}{\sigma}+O\left[(\Delta t)^{3 / 2}\right]\right\}
$$

where again, $\mu$ is a parameter whose meaning will become clear from context. This in turn will mean that the probability of a negative increment in the stock's price will be:

$$
\frac{1}{2}\left\{1-\tanh \left[\frac{\mu \sqrt{x(t) \Delta t}}{\sigma}\right]\right\}=\frac{1}{2}\left\{1-\frac{\mu \sqrt{x(t) \Delta t}}{\sigma}+O\left[(\Delta t)^{3 / 2}\right]\right\}
$$

Bringing these results together shows that the expected increment in the stock's price over the period from time $t$ until time $(t+\Delta t)$ will be: 


$$
\begin{gathered}
E[\Delta x(t)]=\frac{1}{2}\left\{\sigma \sqrt{x(t) \Delta t}+O\left[(\Delta t)^{3 / 2}\right]\right\}\left\{1+\frac{\mu \sqrt{x(t) \Delta t}}{\sigma}+O\left[(\Delta t)^{3 / 2}\right]\right\}+ \\
\frac{1}{2}\left\{-\sigma \sqrt{x(t) \Delta t}+O\left[(\Delta t)^{3 / 2}\right]\right\}\left\{1-\frac{\mu \sqrt{x(t) \Delta t}}{\sigma}+O\left[(\Delta t)^{3 / 2}\right]\right\}
\end{gathered}
$$

or, upon evaluation:

$$
E[\Delta x(t)]=\mu x(t) \Delta t+O\left[(\Delta t)^{3 / 2}\right]
$$

One can then take limits across the above expression in which case it follows that the instantaneous expected increment (per unit time) in the stock's price will be:

$$
\operatorname{Limit}_{\Delta t \rightarrow 0} \frac{E[\Delta x(t)]}{\Delta t}=\frac{E[d x(t)]}{d t}=\mu x(t)
$$

Note, in particular, how this shows that $\mu$ is the expected proportionate change in the stock's price or equivalently, the expected instantaneous buy and hold return on the stock on a per unit time basis. Similar procedures show that the variance of the increment in the stock's price will be:

$$
\begin{gathered}
\operatorname{Var}[\Delta x(t)]=\frac{1}{2}\left\{\sigma \sqrt{x(t) \Delta t}-\mu x(t) \Delta t+O\left[(\Delta t)^{3 / 2}\right]\right\}^{2} \cdot\left\{1+\frac{\mu \sqrt{x(t) \Delta t}}{\sigma}+O\left[(\Delta t)^{3 / 2}\right]\right\}+ \\
\frac{1}{2}\left\{-\sigma \sqrt{x(t) \Delta t}-\mu x(t) \Delta t+O\left[(\Delta t)^{3 / 2}\right]\right\}^{2} \cdot\left\{1-\frac{\mu \sqrt{x(t) \Delta t}}{\sigma}+O\left[(\Delta t)^{3 / 2}\right]\right\}
\end{gathered}
$$

or upon evaluation:

$$
\operatorname{Var}[\Delta x(t)]=\sigma^{2} x(t) \Delta t+O\left[(\Delta t)^{3 / 2}\right]
$$


One can again take limits across the above expression in which case it follows that the instantaneous variance of the increment in the stock's price will be:

$$
\operatorname{Limit}_{\Delta t \rightarrow 0} \frac{\operatorname{Var}[\Delta x(t)]}{\Delta t}=\frac{\operatorname{Var}[d x(t)]}{d t}=\sigma^{2} x(t)
$$

This in turn will mean that the variance of instantaneous increments in the stock's price will be proportional to the current stock price, with $\sigma^{2}$ serving as the constant of proportionality. One can then use this and previous results to define the standardised variable:

$$
q(t) \equiv \frac{d x(t)-E[d x(t)]}{\sqrt{\operatorname{Var}[d x(t)]}}=\frac{d x(t)-\mu x(t) d t}{\sigma \sqrt{x(t)} \sqrt{d t}}
$$

It then follows that the instantaneous increment in the stock's price will be governed by the stochastic differential equation for the Feller diffusion; namely:

$$
d x(t)=\mu x(t) d t+\sigma \sqrt{x(t)} \cdot d q(t)
$$

where $d q(t) \equiv q(t) \cdot \sqrt{d t}$ is a white noise process with unit variance parameter (Hoel, Port and Stone 1987, 142).

Now, one can use the differential equation (8) for the Feller diffusion in conjunction with the Fokker-Planck equation (Cox and Miller 1965, 213-215) to show that the conditional probability density, $p(x(0), x(t), t)$, for the stock's price, $x(t)$, at time $t$ will satisfy the partial differential equation (Feller 1951a, 235):

$$
\frac{1}{2} \sigma^{2} x(t) \frac{\partial^{2} p}{\partial x(t)^{2}}+\left(\sigma^{2}-\mu x(t)\right) \frac{\partial p}{\partial x(t)}-\mu p=\frac{\partial p}{\partial t}
$$


Moreover at $t=0$ the stock's conditional probability density will take the form of a Dirac delta function that is completely concentrated at $x(0)$ (Sneddon 1961, 51-53; Cox and Miller 1965, 209). One can obtain the solution of this initial value problem by first making the substitution (Nariboli 1977, 165):

$$
p(x(0), x(t), t)=v(\xi) \cdot \frac{e^{\mu t}}{\left(e^{\mu t}-1\right)^{2}} \cdot \exp \left\{-\frac{2 \mu x(0)}{\sigma^{2}\left(e^{\mu t}-1\right)}+\frac{2 \mu x(t)}{\sigma^{2}\left(e^{\mu t}-1\right)^{2}}\right\}
$$

where $\xi=\frac{x(t) e^{\mu t}}{\left(e^{\mu t}-1\right)^{2}}$. Differentiation and tedious algebra will then show that under these transformations the Fokker-Planck equation (9) is reduced to the ordinary differential equation:

$$
\frac{1}{2} \sigma^{2} \xi v^{\prime \prime}(\xi)+\left(2 \mu \xi+\sigma^{2}\right) v^{\prime}(\xi)+2 \mu\left(\frac{\mu}{\sigma^{2}} \xi+1-\frac{\mu}{\sigma^{2}} x(0)\right) v(\xi)=0
$$

If one then makes the further substitution:

$$
v(\xi)=\frac{2 \mu}{\sigma^{2}} \cdot \exp \left\{\frac{-2 \mu(\xi+x(0))}{\sigma^{2}}\right\} \sqrt{\frac{x(0)}{\xi}} \cdot G(\xi)
$$

the differential equation (11) becomes:

$$
\frac{1}{2} \sigma^{2} \sqrt{\xi} G^{\prime \prime}(\xi)+\frac{\sigma^{2}}{2 \sqrt{\xi}} G^{\prime}(\xi)-\left\{\frac{\sigma^{2}}{8 \xi \sqrt{\xi}}+\frac{2 \mu^{2}}{\sigma^{2}} \frac{x(0)}{\sqrt{\xi}}\right\} G(\xi)=0
$$

Direct substitution into equation (13) will then show:

$$
G(\xi)=I_{l}\left(2 \sqrt{\frac{2 \mu x(0) \xi}{\sigma^{2}}}\right)=I_{1}\left(\frac{4 \mu}{\sigma^{2}} \cdot \frac{\sqrt{x(0) x(t) e^{\mu t}}}{e^{\mu t}-1}\right)
$$


where $I_{l}(\cdot)$ is the modified Bessel function of the first kind of order one. ${ }^{3}$ It then

follows that the conditional probability density of the stock's price is given by:

$$
p(x(0), x(t), t)=
$$

$$
\frac{2 \mu}{\sigma^{2}} \cdot \exp \left\{\frac{-2 \mu(\xi+x(0))}{\sigma^{2}}\right\rceil \sqrt{\frac{x(0)}{\xi}} \cdot G(\xi) \cdot \frac{e^{\mu t}}{\left(e^{\mu t}-1\right)^{2}} \cdot \exp \left\{-\frac{2 \mu x(0)}{\sigma^{2}\left(e^{\mu t}-1\right)}+\frac{2 \mu x(t)}{\sigma^{2}\left(e^{\mu t}-1\right)^{2}}\right\}
$$

$$
\frac{2 \mu}{\sigma^{2}\left(e^{\mu t}-1\right)} \cdot \sqrt{\frac{x(0) e^{\mu t}}{x(t)}} \cdot \exp \left\{-\frac{2 \mu}{\sigma^{2}} \cdot \frac{x(0) e^{\mu t}+x(t)}{e^{\mu t}-1}\right\} \sum_{j=0}^{\infty} \frac{1}{j !(j+1) !}\left\{\frac{2 \mu}{\sigma^{2}} \cdot \frac{\sqrt{x(0) x(t) e^{\mu t}}}{e^{\mu t}-1}\right\}
$$

Moreover, if one then makes the substitutions:

$$
y=\frac{2 \mu}{\sigma^{2}} \cdot \frac{\sqrt{x(0) x(t) e^{\mu t}}}{e^{\mu t}-1}, d y=\frac{\mu}{\sigma^{2}} \cdot \sqrt{\frac{x(0) e^{\mu t}}{x(t)}} \cdot \frac{d x(t)}{e^{\mu t}-1} \text { and } \quad \gamma=\frac{2 \mu}{\sigma^{2}} \cdot \frac{x(0) e^{\mu t}}{e^{\mu t}-1}
$$

3 In order to obtain this result, one must use the recurrence relationships $\frac{d I_{1}(z)}{d z}=I_{0}(z)-\frac{1}{z} \cdot I_{1}(z)$ and $\frac{d I_{O}(z)}{d z}=I_{1}(z)$, where $I_{0}(z)$ is the modified Bessel function of the first kind of order zero (Abramowitz and Stegun 1964, 376).

${ }^{4}$ Feller (1951a, 235) states this probability density without proof, whilst Feller (1951b, 180) uses the Fokker-Planck equation (9) to determine the Laplace transform of the probability density which he then inverts to determine the probability density itself. Unfortunately, the probability density determined in Feller (1951a, 235) differs from that stated in Feller $(1951 \mathrm{~b}, 180)$ by a multiple of $2 b$ - where $b=\mu$ in our analysis is the expected buy and hold return on the stock on a per unit time basis. It thus follows that the probability density determined in Feller $(1951 b, 180)$ is incorrect - something that is of considerable importance given that the probability density summarised in Feller $(1951 \mathrm{~b}, 180)$ has been widely used in empirical analysis for parameter estimation (Gibbons and Ramaswamy, 1993). 
it follows that the probability density for the Feller diffusion possesses the following canonical representation: ${ }^{5}$

$$
p(x(0), x(t), t)=2 \cdot \exp \left\{-x\left(1+\frac{y^{2}}{\gamma^{2}}\right)\right\} \sum_{j=0}^{\infty} \frac{y^{2 j+1}}{j !(j+1) !}
$$

One can then use this latter result in conjunction with a simple application of the Algebra of Limits to show:

$$
\underset{x(t) \rightarrow 0}{\operatorname{Limit}} p(x(0), x(t), t)=0 \text { and } \underset{x(t) \rightarrow \infty}{\text { Limit }} p(x(0), x(t), t)=0
$$

which are necessary conditions for the existence of convergent moments (Gan and Waxman 2015). We also demonstrate in the Appendix how one can use these results to show that the conditional probability of the stock's price falling away to zero at some unspecified time in the future (that is, the conditional probability of eventual bankruptcy) amounts to $\exp \left\{\frac{-2 \mu x(0)}{\sigma^{2}}\right\}$ (Feller 1951a, 236). ${ }^{6}$

\section{Expected Logarithmic Return}

The conditional expected logarithmic return over the period from time zero until time $t$ on a stock whose price evolves in terms of the Feller diffusion is defined as follows:

5 In the Appendix we use this result to show (Feller 1951a, 236):

$$
\int_{0}^{\infty} p(x(0), x(t), t) d x(t)=1-\exp \left\{-\frac{2 \mu}{\sigma^{2}} \frac{x(0)}{1-e^{-\mu t}}\right\}
$$

Note how this result shows that at $t=0$ the conditional probability density for the Feller diffusion as given by equation (16) will take the form of a Dirac delta function that is completely concentrated at $x(0)$ (Sneddon 1961, 51-53; Cox and Miller 1965, 209).

6 Feller (1951a, 236) again states this result without proof. Cox and Miller (1965, 236-237) provide an alternative proof based on a series expansion of the moment generating function for the probability density (16). 


$$
M(t) \equiv E\left\{\log \left[\frac{x(t)}{x(0)}\right]\right\}=\int_{0}^{\infty} \log \left[\frac{x(t)}{x(0)}\right] p(x(0), x(t), t) d x(t)
$$

Differentiating through this expression and then substituting the Fokker-Planck equation for the Feller diffusion will then show (Cox and Miller 1965, 213-215):

$$
\begin{gathered}
M^{\prime}(t)=\int_{0}^{\infty} \log \left[\frac{x(t)}{x(0)}\right] \cdot \frac{\partial p(x(0), x(t), t)}{\partial t} d x(t)= \\
\int_{0}^{\infty} \log \left[\frac{x(t)}{x(0)}\right]\left\{\frac{\partial^{2}}{\partial x^{2}(t)}\left[\frac{1}{2} \sigma^{2} x(t) p(x(0), x(t), t)\right]-\frac{\partial}{\partial x(t)}[\mu x(t) p(x(0), x(t), t)]\right\} d x(t)
\end{gathered}
$$

Moreover, one can apply integration by parts to the second term on the right hand side of the above expression in which case it follows:

$$
\begin{gathered}
\int_{0}^{\infty} \log \left[\frac{x(t)}{x(0)}\right] \frac{\partial}{\partial x(t)}[\mu x(t) p(x(0), x(t), t)] d x(t)= \\
\left.\left.\log \left[\frac{x(t)}{x(0)}\right][\mu x(t) p(x(0), x(t), t)]\right|_{0} ^{\infty}-\mu \int_{0}^{\infty} p(x(0), x(t), t)\right] d x(t)=-\mu
\end{gathered}
$$

One can also apply repeated integration by parts to the first term in equation (19) in which case we have:

$$
\begin{aligned}
& \int_{0}^{\infty} \log \left[\frac{x(t)}{x(0)}\right] \frac{\partial^{2}}{\partial x^{2}(t)}\left[\frac{1}{2} \sigma^{2} x(t) p(x(0), x(t), t)\right] d x(t)= \\
& \\
& \quad-\int_{0}^{\infty} \frac{1}{x(t)} \cdot \frac{\partial}{\partial x(t)}\left[\frac{1}{2} \sigma^{2} x(t) p(x(0), x(t), t)\right] d x(t)=-\frac{1}{2} \sigma^{2} \int_{0}^{\infty} \frac{p(x(0), x(t), t)}{x(t)} d x(t)
\end{aligned}
$$


Bringing these results together by substituting equation (20) and equation (21) into equation (19) shows that the conditional expected logarithmic return on the stock satisfies the following differential equation:

$$
M^{\prime}(t)=\mu-\frac{1}{2} \sigma^{2} \int_{0}^{\infty} \frac{p(x(0), x(t), t)}{x(t)} d x(t)=\mu-\frac{1}{2} \sigma^{2} \cdot E\left\{\frac{1}{x(t)}\right\}
$$

Moreover, integrating across the above equation and applying the initial condition $M(0)=E\left\{\log \left[\frac{x(0)}{x(0)}\right]\right\}=0$ will then show:

$$
M(t)=E\left\{\log \left[\frac{x(t)}{x(0)}\right]\right\}=\mu t-\frac{1}{2} \sigma^{2} \int_{0}^{t} E\left\{\frac{1}{x(s)}\right\} d s
$$

where $\frac{1}{2} \sigma^{2} \int_{0}^{t} E\left\{\frac{1}{x(s)}\right\} d s>0$ by virtue of the fact that stock prices under the Feller diffusion are strictly non-negative.

Note how this result shows that the conditional expected logarithmic return over the period from time zero until time $t$ will be equal to the expected buy and hold return on the stock over this period, $\mu t$, less a factor which hinges (at least partially) on the volatility parameter, $\sigma$. Moreover, this result possesses a superficial similarity to the expected logarithmic return on a stock whose price evolves in terms of the Geometric Brownian Motion; namely (Karlin and Taylor 1981, 363-364):

$$
E\left\{\log \left[\frac{x(t)}{x(0)}\right]\right\}=\theta t-\frac{1}{2} \delta^{2} t
$$


where $\theta$ is the expected instantaneous buy and hold return (and $\theta t$ is the buy and hold return over the period from time zero until time $t$ ), whilst $\delta^{2}$ is the instantaneous variance of the logarithmic return on the stock (and $\delta^{2} t$ is the variance of the logarithmic return over the period from time zero until time $t$ ). Note how for both the Geometric Brownian Motion and the Feller diffusion the expected logarithmic return is equal to the expected buy and hold return less an adjustment which is related to the volatility of the return. However, for the Geometric Brownian Motion the volatility adjustment, $\frac{1}{2} \delta^{2} t$, is a constant which does not depend the opening stock price, $x(0)$. This is in contrast to the volatility adjustment for the Feller diffusion which varies with the magnitude of the opening stock price and is also related to the probability of the stock's price eventually falling away to zero. One can demonstrate this latter point in more detail by considering the conditional expectation of the following exponential integral: ${ }^{7}$

$$
E\left\{\int_{x(0)}^{x(t)} \frac{\exp \left[\frac{-2 \mu(x(t)-y)}{\sigma^{2}}\right]}{y} d y\right\}=\int_{0}^{\infty} \int_{x(0)}^{x(t)} \frac{\exp \left[\frac{-2 \mu(x(t)-y)}{\sigma^{2}}\right]}{y} \cdot p(x(0), x(t), t) d y d x(t)
$$

where, as previously, $p(x(0), x(t), t)$ is the conditional probability density for the Feller diffusion as given by equation (16) and $\exp \left\{\frac{-2 \mu x(t)}{\sigma^{2}}\right\}$ is the conditional probability of ultimate extinction - that is, the probability that the stock's price will eventually fall away to zero or equivalently, that the firm will enter bankruptcy (Feller 1951a, 236).

\footnotetext{
7 The exponential integral given here arises in a number of areas relating to the Feller diffusion. In the Appendix, for example, we demonstrate how the average time it will take for a stock's price to reach one or other of the upper and lower selling price triggers associated with a filter rule trading strategy (Fama and Blume 1966; Hong and Stein 1999; Sweeney 1988; Chan et al. 1996; D’Aspremont 2011) is stated in terms of the exponential integral given here.
} 
Differentiating through this expression and substituting the Fokker-Planck equation for the Feller diffusion will then show:

$$
\int_{0}^{\infty} \int_{x(0)}^{x(t)} \frac{\exp \left[\frac{-2 \mu(x(t)-y)}{\sigma^{2}}\right]}{y} \cdot \frac{\partial p(x(0), x(t), t)}{\partial t} \cdot d y d x(t)=
$$

$$
\int_{0}^{\infty} \int_{x(0)}^{x(t)} \frac{\exp \left[\frac{-2 \mu(x(t)-y)}{\sigma^{2}}\right]}{y}\left\{\frac{\partial^{2}}{\partial x^{2}(t)}\left[\frac{1}{2} \sigma^{2} x(t) p(x(0), x(t), t)\right]-\frac{\partial}{\partial x(t)}[\mu x(t) p(x(0), x(t), t)]\right\} d y d x(t)
$$

Applying repeated integration by parts to the first term on the right hand side of the above expression implies:

$\frac{1}{2} \sigma^{2} \int_{0}^{\infty}\left\{\exp \left[\frac{-2 \mu x(t)}{\sigma^{2}}\right] \int_{x(0)}^{x(t)} \frac{\exp \left(\frac{2 \mu y}{\sigma^{2}}\right)}{y} d y\right\}\left\{\frac{\partial^{2}}{\partial x^{2}(t)}[x(t) p(x(0), x(t), t)]\right\} d y d x(t)=$

$$
\frac{1}{2} \sigma^{2} \int_{0}^{\infty}\left\{\frac{4 \mu^{2}}{\sigma^{4}} \exp \left[\frac{-2 \mu x(t)}{\sigma^{2}}\right] \int_{x(0)}^{x(t)} \frac{\exp \left(\frac{2 \mu y}{\sigma^{2}}\right)}{y} d y-\frac{1}{x^{2}(t)}-\frac{2 \mu}{\sigma^{2} x(t)}\right\} x(t) p(x(0), x(t), t) d x(t)
$$

A similar application of integration by parts to the second term on the right hand side of equation (25) will also show: 
$\mu \int_{0}^{\infty}\left\{\exp \left[\frac{-2 \mu x(t)}{\sigma^{2}}\right] \int_{x(0)}^{x(t)} \frac{\exp \left(\frac{2 \mu y}{\sigma^{2}}\right)}{y} d y\right\}\left\{\frac{\partial}{\partial x(t)}[\mu x(t) p(x(0), x(t), t)]\right\} d x(t)=$

$$
\int_{0}^{\infty}\left\{\frac{2 \mu^{2}}{\sigma^{2}} \cdot \exp \left[\frac{-2 \mu x(t)}{\sigma^{2}}\right] \int_{x(0)}^{x(t)} \frac{\exp \left(\frac{2 \mu y}{\sigma^{2}}\right)}{y} d y-\frac{\mu}{x(t)}\right\} x(t) p(x(0), x(t), t) d x(t)
$$

Substituting these latter two results into equation (25) will then imply:

$$
E\left\{\int_{x(0)}^{x(t)} \frac{\exp \left[\frac{-2 \mu(x(t)-y)}{\sigma^{2}}\right]}{y} d y\right\}=
$$

$$
\int_{0}^{\infty} \int_{x(0)}^{x(t)} \frac{\exp \left[\frac{-2 \mu(x(t)-y)}{\sigma^{2}}\right]}{y} \cdot p(x(0), x(t), t) d y d x(t)=-\frac{1}{2} \sigma^{2} \int_{0}^{t} E\left\{\frac{1}{x(s)}\right\} d s
$$

Taking equation (26) in conjunction with equation (22) will then mean that the conditional expected logarithmic return over the period from time zero until time $t$ on a stock whose price evolves in terms of the Feller diffusion may be expressed as: ${ }^{8}$

${ }^{8}$ Here it is important to note that a simple application of L'Hôpital's rule shows:

$$
\int_{x(0)}^{x(t)} \frac{\exp \left\{\frac{2 \mu}{\sigma^{2}}(x(t)-y)\right\}}{y} d y=\frac{\int_{x(0)}^{x(t)} \frac{\exp \left(\frac{2 \mu y}{\sigma^{2}}\right)}{y} d y}{\exp \left(\frac{2 \mu x(t)}{\sigma^{2}}\right)} \rightarrow \frac{1}{\frac{2 \mu}{\sigma^{2}} x(t)} \rightarrow 0
$$

as $x(t) \rightarrow \infty$ - a result which is consistent with the numerical example summarised in Table 1 below. 


$$
E\left\{\log \left[\frac{x(t)}{x(0)}\right]\right\}=\mu t+E\left\{\int_{x(0)}^{x(t)} \frac{\exp \left[\frac{-2 \mu(x(t)-y)}{\sigma^{2}}\right]}{y} d y\right\}
$$

One can demonstrate the implementation of this result by considering a stock with an expected instantaneous buy and hold return of $\mu=0.15$ (that is, $15 \%$ per annum) and a volatility parameter of $\sigma=1$. Substituting these parameter values into equation (27) will then show that the conditional expected annual logarithmic return on the stock will be:

$$
E\left\{\log \left[\frac{x(1)}{x(0)}\right]\right\}=0.15+E\left\{\int_{x(0)}^{x(1)} \frac{\exp (-0.3(x(1)-y))}{y} d y\right\}
$$

Table 1 summarises the conditional expected annual logarithmic return on a stock with the above parameter values (that is, $\mu=0.15$ and $\sigma=1$ ) for various values of the opening stock price, $x(0) .{ }^{9}$ Thus, when the opening stock price amounts to $x(0)=£ 5$ then the conditional expected logarithmic return is $4.65 \%$ per annum reflecting the fact that the

\section{TABLE ONE ABOUT HERE}

probability of ultimate extinction (that is, the probability the firm will eventually enter bankruptcy) amounts to (Feller 1951a, 236):

$$
\exp \left[\frac{-2 \mu x(0)}{\sigma^{2}}\right]=\exp \left[\frac{-2 \times 0.15 \times 5}{1^{2}}\right]=0.2231
$$

\footnotetext{
${ }^{9}$ All integrals associated with the Feller diffusion are evaluated numerically using a combination of $n=50$ point Gauss-Legendre quadrature and $n=50$ point Gauss-Laguerre quadrature. These quadrature rules integrate polynomial expressions of order $(2 n+1)=101$ or less exactly (Carnahan, Luther and Wilkes 1969, 101-105).
} 
or, just over $22 \%$. However, as the opening stock price increases in magnitude and the probability of extinction declines, then the conditional expected logarithmic return on the stock increases significantly. Thus, when the opening stock price is $x(0)=£ 90$, there is only a trivial probability of ultimate extinction and the conditional expected logarithmic return on the stock has grown to $14.48 \%$ per annum. ${ }^{10}$ Hence, the results summarised in this table show how the conditional expected logarithmic return on a stock whose price evolves in terms of the Feller diffusion is not an intertemporal constant but varies according to the conditional probability of ultimate extinction. This is in direct contrast to the Geometric Brownian Motion (which underscores much of the empirical work conducted in the discipline), for which the conditional expected annual logarithmic return will be the same irrespective of the opening stock price, $x(0)$. This in turn will mean that if a researcher mistakenly assumes a stock's price evolves in terms of a Geometric Brownian Motion when, in fact, the actual process describing the evolution of the stock's price is the Feller diffusion, there will be systematic biases in the abnormal returns calculated by the researcher. ${ }^{11}$

10 One can reinforce this point by using equation (8) in conjunction with Itô's formula to determine the distributional properties of instantaneous increments in the logarithmic return, $r(t)=\log \left[\frac{x(t)}{x(0)}\right]$. It then follows:

$$
d r(t)=\frac{\partial r}{\partial x(t)} \cdot d x(t)+\frac{1}{2} \cdot \frac{\partial^{2} r}{\partial x(t)^{2}} \cdot[d x(t)]^{2}=\frac{d x(t)}{x(t)}-\frac{1}{2} \cdot\left[\frac{d x(t)}{x(t)}\right]^{2}=\left(\mu-\frac{1}{2} \cdot \frac{\sigma^{2}}{x(t)}\right) d t+\frac{\sigma}{\sqrt{x(t)}} \cdot d q(t)
$$

This result shows that the instantaneous increment in the logarithmic return (per unit time) will have a mean of $\frac{E[d r(t)]}{d t}=\left(\mu-\frac{1}{2} \cdot \frac{\sigma^{2}}{x(t)}\right)$ and variance $\frac{\operatorname{Var}[d r(t)]}{d t}=\frac{\sigma^{2}}{x(t)}$. Note that when $x(t)<\frac{\sigma^{2}}{2 \mu}$ or equivalently, the probability of eventual extinction exceeds $e^{-1} \approx 0.367879$, then the expected instantaneous change in the stock's logarithmic return will be negative. This in turn will mean that in expectations there will be a downward spiral in the logarithmic return that will culminate ultimately, in the firm entering bankruptcy (that is, ultimate extinction).

11 Further insight may be obtained into the biases which arise from mistakenly assuming a stock's price, $x(t)$, evolves in terms of a Geometric Brownian Motion by applying a Taylor series expansion to $\log [x(t)]$ about the point $x(0) e^{\mu t}$. If one then takes expectations across the series expansion and substitutes the first four central moments for the Feller diffusion (Davidson and Tippett 2012, 218 and 232) one will end up with the following series expansion for the expected logarithmic return for the Feller diffusion: 
This result has important implications for empirical analysis. There is, for example, an extensive body of empirical literature that documents evidence of a long-run positive (negative) price drift after positive (negative) news announcements. This drift in abnormal returns has been identified following earnings announcements (Ball and Brown, 1968; Bernard and Thomas, 1989), stock splits (Grinblatt et al., 1984), share repurchases (Lakonishok and Vermaelen, 1990), dividend initiations and omissions (Michaely et al., 1995) and revisions to analysts' earnings forecasts (Elton et al., 1984). As this post-announcement drift in abnormal returns is difficult to reconcile with models of rational expectations, the prevailing view in the literature is that these empirical observations can be attributed to investor under-reaction (Fama, 1998). ${ }^{12}$

It should be noted that a drift pattern in abnormal returns can also occur if a stock's benchmark return is based on the Geometric Brownian Motion when in fact the actual process describing the evolution of the stock's price is the Feller diffusion. Here it will be recalled that when a stock is associated with positive (negative) news, there will in general be an instantaneous positive (negative) increase in the market price of the given stock. Moreover, under the assumption that the stock's price evolves in terms of the Geometric Brownian Motion the instantaneous price shock would have no impact on the

$$
E\left\{\log \left[\frac{x(t)}{x(0)}\right]\right\}=\mu t-\frac{1}{2} \frac{\sigma^{2}}{\mu x(0)}\left(1-e^{-\mu t}\right)-\frac{1}{4} \frac{\sigma^{4}}{\mu^{2} x^{2}(0)}\left(1-e^{-\mu t}\right)^{2}-\frac{3}{8} \frac{\sigma^{6}}{\mu^{3} x^{3}(0)}\left(1-e^{-\mu t}\right)^{3}+
$$

Substituting $\mu=0.15, \sigma=1$ and $t=1$ shows that the above expansion exactly replicates the results summarised in Table 1 except when $x(0)=\$ 5$ and $x(0)=\$ 3^{1} / 3$ where the expansion gives expected logarithmic returns of 0.0461 and -0.0168 , respectively. In these latter two cases the series expansion will need to encompass additional terms involving the higher order (fifth, sixth, etc.) moments if it is to give a more satisfactory approximation to the expected logarithmic return. This contrasts with the Geometric Brownian Motion where equation (23) shows that only the first two moments are necessary to give an exact representation of the expected logarithmic return. Moreover, the above series expansion shows that the opening stock price, $x(0)$, affects the expected logarithmic return for the Feller diffusion in a way that is entirely absent from the expected logarithmic return for the Geometric Brownian Motion.

12 A little reflection will convince the reader that our analysis has important implications for a much wider class of issues - including filter trading rules and momentum in stock returns, the prediction of corporate failure and the pricing of derivative securities (Cox 1996). 
benchmark return used to isolate the abnormal returns on the stock. However, as shown in Table 1, if the stock's price evolves in terms of the Feller diffusion, the positive price shock would also result in an increase in the expected return on the stock. Under these circumstances, mistakenly assuming the stock's price evolves in terms of a Geometric Brownian Motion will give the appearance of a post-announcement drift in the abnormal returns that accrue on the stock. ${ }^{13}$

Whilst we make no contention about the actual source of post-announcement drift anomalies the results summarised in Table 1 are informative as they identify an alternative set of conditions under which an empirical researcher may identify a postevent drift in stock prices. We would not contend either that the Geometric Brownian Motion or the Feller diffusion is capable of providing a completely satisfactory description of the way stock prices evolve in practice. It nonetheless warrants emphasising that if two commonly implemented stochastic processes can lead to such conflicting prescriptions for the calculation of abnormal returns, then it is important for researchers (and others) to assess the compatibility of the empirically observed returns on a given stock (or portfolio) with the assumed distributional assumptions on which the calculation of abnormal returns are based. We note that such goodness of fit testing

\footnotetext{
13 Here it will be recalled that the expected logarithmic return on the Geometric Brownian Motion is a constant which is independent of the opening stock price, $x(0)$. Whether the stock price is "low" or "high", the expected logarithmic return is the same. This in turn means that the expected logarithmic returns in Table 1 under the false assumption of the Geometric Brownian Motion will be the same irrespective of the opening stock price. One can demonstrate the nature of the biases which arise under the false assumption of the Geometric Brownian Motion by supposing a researcher undertakes parameter estimation when the stock's price is concentrated around $x(0)=£ 45$ per share. Consistent with the results summarised in Table 1 the researcher will find that the average annual logarithmic return over the estimation period will be around $13.96 \%$ (per annum). Given this, the researcher will set this return as the "benchmark" against which the stock's subsequent performance is to be assessed. However, if the researcher applies this benchmark to returns based on opening stock prices that are not in the neighbourhood of $x(0)=£ 45$, there will be evidence of (fictitious) abnormal returns. Thus, for example, Table 1 shows that if the stock's price is in the neighbourhood of $x(0)=£ 30$ then applying a benchmark return of $13.96 \%$ will lead, on average, to a negative abnormal return of around $(0.1343-0.1396=)-0.53 \%$ (per annum). Similarly, if the stock's price is in the vicinity of $x(0)=£ 60$, there will, on average, be a positive abnormal return of around $(0.1422-0.1396=) 0.26 \%$ (per annum).
} 
procedures have rarely been conducted in the empirical literature of the discipline and where they have, it is seldom the case that the empirically calculated returns are compatible with the Gaussian probability density on which most of the commonly employed abnormal returns quadrature rules are based.

\section{Conclusions}

We determine the conditional expected logarithmic return on a stock whose price evolves in terms of the Feller (1951a) diffusion and then use it to demonstrate how one must know the exact stochastic process that generates a stock's return before one can determine the correct way to calculate the abnormal returns which accrue on the stock. We demonstrate this by considering a stock whose price actually evolves in terms of the Feller diffusion but where a researcher mistakenly assumes the stock's price develops in terms of the more commonly employed Geometric Brownian Motion. We show that when abnormal returns are calculated under the mistaken belief that the stock's price evolves in terms of the Geometric Brownian Motion, there will be the appearance of abnormal returns when, in fact, there are no abnormal returns at all. This occurs because the expected logarithmic return on a stock whose price evolves in terms of the Feller diffusion varies according to the conditional probability of ultimate extinction (that is, the probability that the stock's price will eventually fall away zero or equivalently, the firm will enter bankruptcy). This is in contrast with the Geometric Brownian Motion for which the conditional expected logarithmic return is the same irrespective of the current level of the stock's price. Our analysis thus suggests that it is crucially important for researchers to test the compatibility of the empirically observed returns on a given stock with the assumed distributional assumptions on which the calculation of abnormal returns 
are based before deciding on an appropriate quadrature formula through which to isolate the abnormal returns that accrue on the stock. 


\section{Appendix: Probability Density of the Feller Diffusion}

One can integrate through the probability density given by equation (17) of the text, over the semi-infinite real line and thereby show:

$$
2 \int_{0}^{\infty} \exp \left\{-\chi\left(1+\frac{y^{2}}{\gamma^{2}}\right)\right\} \cdot \sum_{j=0}^{\infty} \frac{y^{(2 j+1)}}{j !(j+1) !} d y=2 e^{-\gamma} \cdot \sum_{j=0}^{\infty} \frac{1}{j !(j+1) !} \int_{0}^{\infty} \exp \left(-\gamma^{-1} y^{2}\right) y^{2 j+1} d y
$$

Here one can let $z=\gamma^{-1} y^{2}$ from which it follows $\frac{1}{2} \gamma d z=y d y$. Substitution will then show:

$$
2 \int_{0}^{\infty}\left(\exp \left\{-\gamma\left(1+\frac{y^{2}}{\gamma^{2}}\right)\right\} \cdot \sum_{j=0}^{\infty} \frac{y^{(2 j+1)}}{j !(j+1) !} d y=e^{-\gamma} \cdot \sum_{j=0}^{\infty} \frac{\gamma^{j+1}}{j !(j+1) !} \int_{0}^{\infty} z^{j} e^{-z} d z=e^{-\gamma} \cdot \sum_{j=0}^{\infty} \frac{\gamma^{j+1}}{(j+1) !}=1-e^{-\gamma}\right.
$$

It then follows that the probability of the stock price falls away to nothing before time $t$ is (Feller 1951a, 236):

$$
1-2 \int_{0}^{\infty} \exp \left\{-x\left(1+\frac{y^{2}}{\gamma^{2}}\right)\right\} \cdot \sum_{j=0}^{\infty} \frac{y^{(2 j+1)}}{j !(j+1) !} d y=e^{-\gamma}=\exp \left\{-\frac{2 \mu}{\sigma^{2}} \cdot \frac{x(0)}{1-e^{-\mu t}}\right\}
$$

Moreover, this result also shows that the probability of the stock's price falling away to zero at some unspecified future time (that is, the probability of ultimate extinction) amounts to (Feller 1951a, 236):

$$
\operatorname{Limit}_{t \rightarrow \infty} \exp \left\{-\frac{2 \mu}{\sigma^{2}} \cdot \frac{x(0)}{1-e^{-\mu t}}\right\}=\exp \left\{-\frac{2 \mu x(0)}{\sigma^{2}}\right\}
$$

\section{Appendix: Filter Rule Trading Strategy}

Consider a simple filter rule trading strategy under which a stock is purchased at a price of $x$ at time zero (Fama and Blume 1966; Hong and Stein 1999; Sweeney 1988; Chan et al. 1996; D'Aspremont 2011). If the stock's price subsequently rises to $b \geq x$ then the 
stock is sold and the profits realised. Alternatively, if the stock's price falls to $a \leq x$ before it rises to $b \geq x$ then the investor invokes a "stop loss" strategy of selling the stock and realising the losses. It then follows that the probability, $u(x)$, of the stock's price rising from its current level, $x$, to the upper price selling trigger, $b \geq x$, before it falls back to the lower price selling trigger, $a \leq x$, can be determined as the solution of the following differential equation (Cox and Miller 1965, 231):

$$
\frac{1}{2} \sigma^{2} x \cdot \frac{d^{2} u(x)}{d x^{2}}+\mu x \cdot \frac{d u(x)}{d x}=0
$$

with the boundary conditions being $u(a)=0$ and $u(b)=1$. Determining the solution to the above boundary value problem shows:

$$
u(x)=\frac{\exp \left(\frac{-2 \mu x}{\sigma^{2}}\right)-\exp \left(\frac{-2 \mu a}{\sigma^{2}}\right)}{\exp \left(\frac{-2 \mu b}{\sigma^{2}}\right)-\exp \left(\frac{-2 \mu a}{\sigma^{2}}\right)}
$$

This in turn will mean that the probability of the stock's price falling to $a \leq x$ before it rises to $b \geq x$ will be:

$$
1-u(x)=\frac{\exp \left(\frac{-2 \mu b}{\sigma^{2}}\right)-\exp \left(\frac{-2 \mu x}{\sigma^{2}}\right)}{\exp \left(\frac{-2 \mu b}{\sigma^{2}}\right)-\exp \left(\frac{-2 \mu a}{\sigma^{2}}\right)}
$$

Moreover, the average time, $v(x)$, it will take the stock's price to reach one or other of the two (that is, upper and lower) selling price triggers can be determined as the solution of the following differential equation (Cox and Miller 1965, 232):

$$
\frac{1}{2} \sigma^{2} x \cdot \frac{d^{2} v(x)}{d x^{2}}+\mu x \cdot \frac{d v(x)}{d x}=-1
$$


with the boundary conditions being $v(a)=0=v(b)$. Solving the given boundary value problem shows that the average time it will take for the stock's price to rise to $b$ or alternatively, to fall back to $a$ amounts to:

$$
v(x)=\frac{E\left\{\log \left[\frac{x(t)}{x(0)}\right]\right\}-E\left\{\int_{x(0)}^{x(t)} \frac{\exp \left[\frac{-2 \mu(x(t)-y)}{\sigma^{2}}\right]}{y} d y\right\}}{\mu}
$$

where:

$$
E\left\{\log \left[\frac{x(t)}{x(0)}\right]\right\}=u(x) \cdot \log \left(\frac{b}{x}\right)+(1-u(x)) \cdot \log \left(\frac{a}{x}\right)
$$

and:

$$
E\left\{\int_{x(0)}^{x(t)} \frac{\exp \left[\frac{-2 \mu(x(t)-y)}{\sigma^{2}}\right]}{y} d y\right\}=u(x) \int_{x}^{b} \frac{\exp \left[\frac{-2 \mu(b-y)}{\sigma^{2}}\right]}{y} d y+(1-u(x)) \int_{x}^{a} \frac{\exp \left[\frac{-2 \mu(a-y)}{\sigma^{2}}\right]}{y} d y
$$

Now, from equation (27) of the text it follows that the conditional abnormal logarithmic return over the period from time zero until time $t$ on a stock whose price evolves in terms of the Feller diffusion will amount to:

$$
\log \left[\frac{x(t)}{x(0)}\right]-\mu t-E\left\{\int_{x(0)}^{x(t)} \frac{\exp \left[\frac{-2 \mu(x(t)-y)}{\sigma^{2}}\right]}{y} d y\right\}
$$


Hence, comparing equation (27) of the text with equation (B5) above shows that the expected abnormal logarithmic return over the average period, $v(x)$, it will take for the stock's price to either rise to the upper selling price trigger, $b$, or alternatively, fall back to the lower selling price trigger, $a$, turns out to be zero - something one would expect given that the stock's price evolves in terms of a purely random process. ${ }^{14}$

14 Here it is important to emphasise that the expected abnormal buy and hold return under the filter rule trading strategy considered in this section does not turn out to be zero. 
Table 1. Conditional expected annual logarithmic return on a stock whose price evolves in terms of the Feller diffusion with an instantaneous expected buy and hold return of $\mu=0.15$ and volatility parameter of $\sigma=1$.

\begin{tabular}{ccccc} 
& \multicolumn{5}{c}{$\mathbf{x}(\mathbf{1})$} & & \\
$\mathbf{x}(\mathbf{0})$ & $\boldsymbol{E}\left\{\int_{\mathbf{x}(\mathbf{0})}^{\mathbf{e x p}[-\mathbf{0 . 3}(\mathbf{x}(\mathbf{1})-\mathbf{y})]} \mathbf{d y}\right\}$ & $\mathbf{E}\left\{\mathbf{l o g}\left[\frac{\mathbf{x}(\mathbf{1})}{\mathbf{x}(\mathbf{0})}\right]\right\}$ & $\begin{array}{c}\text { Probability } \\
\mathbf{o f} \\
\text { Bankruptcy }\end{array}$ \\
$\frac{\sigma^{2}}{2 \mu}=\frac{10}{3}$ & & & & \\
5 & 0.15 & -0.1605 & -0.0105 & 0.3679 \\
10 & 0.15 & -0.1035 & 0.0465 & 0.2231 \\
15 & 0.15 & -0.0488 & 0.1012 & 0.0498 \\
20 & 0.15 & -0.0320 & 0.1180 & 0.0111 \\
25 & 0.15 & -0.0238 & 0.1262 & 0.0025 \\
30 & 0.15 & -0.0189 & 0.1311 & 0.0006 \\
35 & 0.15 & -0.0157 & 0.1343 & 0.0001 \\
40 & 0.15 & -0.0134 & 0.1366 & 0.0000 \\
45 & 0.15 & -0.0117 & 0.1383 & 0.0000 \\
50 & 0.15 & -0.0104 & 0.1396 & 0.0000 \\
55 & 0.15 & -0.0094 & 0.1406 & 0.0000 \\
60 & 0.15 & -0.0085 & 0.1415 & 0.0000 \\
65 & 0.15 & -0.0078 & 0.1422 & 0.0000 \\
70 & 0.15 & -0.0072 & 0.1428 & 0.0000 \\
75 & 0.15 & -0.0067 & 0.1433 & 0.0000 \\
80 & 0.15 & -0.0062 & 0.1438 & 0.0000 \\
85 & 0.15 & -0.0058 & 0.1442 & 0.0000 \\
90 & 0.15 & -0.0055 & 0.1445 & 0.0000 \\
& & -0.0052 & 0.1448 & 0.0000 \\
& & & &
\end{tabular}

Notes: This table determines the expected annual logarithmic return as defined by equation (27) of the text for a stock with an opening price of $x(0)$ and whose terminal price, $x(1)$, evolves in terms of the Feller diffusion with parameter values of $\mu=0.15$ and $\sigma=1$, respectively. The integral expression summarised in column 3 is evaluated using 50 point Gauss-Legendre quadrature whilst the expectation of the integral expression is evaluated using 50 point Gauss-Laguerre quadrature (Carnahan, Luther and Wilkes 1969, 101-105). The probability of eventual extinction (that is, the probability of bankruptcy) appearing in the final column of the table is computed using equation (A4) in the Appendix. 


\section{References}

Abramowitz, M. and Stegun, I., 1964. Handbook of mathematical functions with formulas, graphs, and mathematical tables. Washington, DC: NBS Applied Mathematics Series 55, National Bureau of Standards.

Ashton, D., Cooke, T. and Tippett, M., 2003. An aggregation theorem for the valuation of equity under linear information dynamics. Journal of Business Finance and Accounting, 30 (3\&4), 413-440.

Ball, R. and Brown. P., 1968. An empirical evaluation of accounting income numbers. Journal of Accounting Research, 6(2), 159-178.

Bernard, V. and Thomas, J., 1989. Post-earnings announcement drift: Delayed price response or risk premium. Journal of Accounting Research. 27(Supplement), 1-36.

Carnahan, B. Luther, H and Wilkes, J., 1969. J., Applied Numerical Methods. (New York: Wiley).

Chan L., Jegadeesh, N. and Lakonishok, J., 1996. Momentum strategies. Journal of Finance, 1996, 51(5), 1681-1713.

Cox, D. and Miller, H., 1965. Theory of stochastic processes. (London: Chapman and Hall).

Cox, J., 1996. The constant elasticity of variance option pricing model. Journal of Portfolio Management. Special Issue, 15-17.

Cox, J. and Ross, S., 1976. The valuation of options for alternative stochastic processes. Journal of Financial Economics, 3(1\&2), 145-166.

D'Aspremont A., 2011. Identifying small mean reverting portfolios, Quantitative Finance, 11(3), 351-364.

Davidson, I. and Tippett, M., 2012. Principles of equity valuation. (Abingdon, Oxon: Routledge).

Elton, E., Gruber, M. and Gultekin, M., 1984. Professional expectations: accurary and diagnosis of errors. Journal of Financial and Quantitative Analysis. 19(4), 351-363.

Fama, E., 1988. Market efficiency, long-term returns, and behavioral finance. Journal of Financial Economics. 49(3), 283-306.

Feller, W., 1951a. Diffusion processes in genetics. Proceedings of the Second Berkley Symposium on Mathematical Statistics and Probability. 54(1), 227-246.

Feller, W., 1951b. Two singular diffusion processes." Annals of Mathematics. 173182. 
Gan, X. and Waxman, D., 2015. Singular solution of the Feller diffusion equation via a spectral decomposition. Physical Review E. 91(1),

Gibbons, M. and Ramaswamy, K., 1993. A test of the Cox, Ingersoll and Ross model of the term structure. Review of Financial Studies. 6(3), 619-658.

Grinblatt, M., Masulis, R. and Titman, S., 1984. The valuation effects of stock splits and stock dividends. Journal of Financial Economics. 13(4), 461-490.

Hoel, P., S. Port, and C. Stone. 1987. Introduction to Stochastic Processes. Long Grove, IL:Waveland Press.

Hong H. and Stein, J., 1999. A unified theory of underreaction, momentum trading, and overreaction in asset markets. Journal of Finance, 54(6), 2143-2184.

Karlin, S. and Taylor, H., 1981. A Second Course in Stochastic Processes. (London: Academic Press).

Lakonishok, J. and Vermaelen, T., 1990. Anomalous price behavior around repurchase tender offers. Journal of Finance. 45(2), 455-477.

Michaely, R., Thaler, R. and Womack, K., 1995. Price reaction to dividend initiations and omissions: overreaction or drift. Journal of Finance. 50(2), 573-608.

Nariboli, G., 1977. Group invariant solutions of the Fokker-Planck equation. Stochastic Processes and their Applications. 5(2), 157-171.

Nelson, D. and Ramaswamy, K., 1990. Simple binomial processes as diffusion approximations in financial models. Review of Financial Studies, 3(3), 393-430.

Sneddon, I. 1961. Fourier Series. London: Routledge \& Kegan Paul Ltd.

Sweeney, R., 1988. Some new filter rule tests: methods and results. Journal of Financial and Quantitative Analysis. 23(3), 285-300. 